\title{
Connection between slow and fast dynamics of molecular liquids around the glass transition
}

Niss, Kristine; Dalle-Ferrier, Cecile; Frick, Bernhard; Russo, Daniela; Dyre, J. C.; AlbaSimionesco, Christiane

Published in:

Physical Review E (Statistical, Nonlinear, and Soft Matter Physics)

DOI:

10.1103/PhysRevE.82.021508

Publication date:

2010

Document Version

Publisher's PDF, also known as Version of record

Citation for published version (APA):

Niss, K., Dalle-Ferrier, C., Frick, B., Russo, D., Dyre, J. C., \& Alba-Simionesco, C. (2010). Connection between slow and fast dynamics of molecular liquids around the glass transition. Physical Review E (Statistical, Nonlinear, and Soft Matter Physics), 82(021508). https://doi.org/10.1103/PhysRevE.82.021508

\section{General rights}

Copyright and moral rights for the publications made accessible in the public portal are retained by the authors and/or other copyright owners and it is a condition of accessing publications that users recognise and abide by the legal requirements associated with these rights.

- Users may download and print one copy of any publication from the public portal for the purpose of private study or research.

- You may not further distribute the material or use it for any profit-making activity or commercial gain.

- You may freely distribute the URL identifying the publication in the public portal.

\section{Take down policy}

If you believe that this document breaches copyright please contact rucforsk@kb.dk providing details, and we will remove access to the work immediately and investigate your claim. 


\title{
Connection between slow and fast dynamics of molecular liquids around the glass transition
}

\author{
Kristine Niss, ${ }^{1,2}$ Cécile Dalle-Ferrier, ${ }^{1}$ Bernhard Frick, ${ }^{3}$ Daniela Russo, ${ }^{3}$ Jeppe Dyre, ${ }^{2}$ and Christiane Alba-Simionesco ${ }^{1,4}$ \\ ${ }^{1}$ Laboratoire de Chimie Physique, Bâtiment 349, Université Paris-Sud, F-91405 Orsay, France \\ ${ }^{2}$ DNRF Centre "Glass and Time," IMFUFA, Department of Sciences, Roskilde University, Postbox 260, DK-4000 Roskilde, Denmark \\ ${ }^{3}$ Institut Laue-Langevin, F-38042 Grenoble, France \\ ${ }^{4}$ Laboratoire Léon Brillouin, UMR 12, CEA-CNRS, 91191-Gif-sur-Yvette, France
}

(Received 14 October 2009; revised manuscript received 1 June 2010; published 19 August 2010)

\begin{abstract}
The mean-square displacement (MSD) was measured by neutron scattering at various temperatures and pressures for a number of molecular glass-forming liquids. The MSD is invariant along the glass-transition line at the pressure studied, thus establishing an "intrinsic" Lindemann criterion for any given liquid. A one-to-one connection between the MSD's temperature dependence and the liquid's fragility is found when the MSD is evaluated on a time scale of $\sim 4 \mathrm{~ns}$, but does not hold when the MSD is evaluated at shorter times. The findings are discussed in terms of the elastic model and the role of relaxations, and the correlations between slow and fast dynamics are addressed.
\end{abstract}

DOI: 10.1103/PhysRevE.82.021508

PACS number(s): 64.70.pm, 25.40.Fq, 63.50.Lm

\section{INTRODUCTION}

A major challenge to current condensed-matter physics is to explain properties of supercooled liquids approaching the glass transition. In particular, there is still no consensus about what causes the dramatic "super-Arrhenius" increase of the liquid's viscosity. The viscosity will for so-called fragile liquids close to the glass transition increase with about one decade (in some cases even more) for a temperature decrease of just $1 \%$. The most popular model is the Adam-Gibbs entropy model [1] which relates the slowing down to an underlying phase transition; this model involves the notion of a growing length scale as the liquid is cooled toward the glass transition. The experimental search for growing length scales uses many different observables, it has been going on for several decades $[2,3]$ and it is an ongoing active field of research [4-6], but there is still no clear conclusion. Another approach is advocated by the so-called elastic models, the main ideas of which date back to a paper by Eyring and co-workers from 1943 [7] and Nemilov's further work in 1968 [8]. These models relate the activation energy to the viscous liquid's short-time elastic properties $[9,10]$. Thus, apparently paradoxical, properties on the pico- or nanosecond time scale could determine the slow molecular relaxations taking place over minutes or hours in the liquid close to the conventional glass-transition temperature. This paper investigates this intriguing prediction by studying several liquids, some under varying pressure, by means of quasielastic neutron scattering experiments providing the most direct measurement available of the mean-square displacement on several short-time scales.

The idea of a connection between the dynamics on time scales differing by ten or more orders of magnitude has also been put forward in other contexts. If correct, it emphasizes the fact that the glass-transition phenomenon involves an exceedingly large dynamical range- and that a full understanding of the glass transition must encompass both fast and slow dynamics. Before proceeding to describe the experiment, we give examples of the ideas and results which point in this direction.
In 1992 it was observed by Buchenau and Zorn [11] that there is a relation between the temperature dependence of the structural relaxation time and the temperature dependence of the mean-square displacement (MSD) observed on the nanopico second time scale in selenium as determined by neutron scattering experiments. Since then, other groups found similar results, showing qualitatively that the larger the fragility is, the stronger is the temperature dependence of the MSD [12-18]. Recent theoretical work relates the mean-square displacement and the vibrational entropy and relates both quantities to the slow dynamics [19]. In 1987 Hall and Wolynes [20] theoretically discussed how the mean-square vibrational displacement controls the relaxation time according to the expression $\tau \propto \exp \left(\right.$ const. $\left./\left\langle u^{2}\right\rangle\right)$. Their approach was later developed into the random first-order transition theory (RFOT) of the glass transition $[21,22]$ where a variational density profile built of Gaussian vibrational displacements around aperiodic atomic positions is optimized for free-energy minimization. Thus the vibrational short-time displacement is the crucial quantity for the RFOT, which was later developed into a full-fledged theory leading to a generalization of the Adam-Gibbs prediction for the relaxation time in terms of the configurational entropy [1].

In more recent works $[23,24]$ Novikov and Sokolov demonstrated a surprising connection between the "fragility"-a measure of how fast the liquid's viscosity (or relaxation time) increases as temperature decreases and enters the glassy state-and elastic properties of the glass: the more fragile the liquid is, the higher is the ratio between the bulk and shear moduli of the resulting glass. However, a study of a larger set of liquids shows that the correlation does not hold in general [25]. Novikov and Sokolov discussed a possible explanation of their correlation in terms of elastic models like the shoving model [9]. This does not seem to be a correct connection because recent experimental work by Nelson and co-workers supports the shoving model while it lends no support to Novikov's and Sokolov's correlation [26]. Sokolov earlier introduced a parameter derived from the measured dynamic structure factor, which relates the strength of the quasielastic scattering intensity at $T_{g}$ normalized to the intensity of the boson peak and the fragility [27]. 
This correlation has also been questioned [28] even if it holds on a very qualitative level (intense boson peaks are seen in strong systems like oxide glasses whereas the boson peak in van der Waals systems is less pronounced). It also appears that this correlation could be more related to isochoric than isobaric fragility [29]. Along similar lines Scopigno and co-workers suggested a correlation between the temperature dependence of the nonergodicity factor measured in the glass and the fragility of the liquid [30,31]. The main point to be noted here is that the two Novikov-Sokolov correlations as well as the Scopigno correlation provide an intriguing connection between short- and long-time properties of the liquid (since the liquid's short-time mechanical properties are those of the glass corresponding to the liquid structure at the temperature in question).

Widmer and Harrowell [32] proposed studying the Maxwell-Boltzmann ensemble averaged mean-square displacement of a particle for any given initial configuration (the "iso-configurational ensemble"), terming this quantity the dynamic propensity of the particle in question. This property's distribution reflects the dynamic heterogeneity of the liquid. Thus, once again, a connection is established between the long-time dynamic properties and MSD on time scales much shorter than the relaxation time.

Leporini and collaborators [33] argued from simulations that there is a universal correlation between the structural relaxation time and the "rattling amplitude" from high- to low-viscosity states. According to this picture the glass softens when the rattling amplitude exceeds a critical value. This implies a "universal" Lindemann criterion for the glass transition, i.e., that the glass transition takes place when the MSD reaches a certain value (see e.g., Refs. [10,34]).

At first sight it appears very surprising that there could be any relation between the alpha-relaxation process-taking place on the second or hour time scale-and mean-square displacements taking place on the nano-/picosecond time scale. It should be recalled, however, that whereas the alpha relaxation is very slow, the barrier transitions themselves are fast. This fact is the starting point for the elastic models. In these models the relation between mean-square displacement and fragility comes very natural, and the stiffness of the material or, equivalently, the steepness of the energy minima, determines the activation energy of the alpha process $[7,10,20]$.

In terms of the vibrational MSD $\left\langle u^{2}\right\rangle$, in the simplest version where the instantaneous bulk and shear moduli are proportional in their temperature variation, all elastic models imply for the activation energy $\Delta E(T) \propto T a^{2} /\left\langle u^{2}\right\rangle(T)$, where $a$ is the average intermolecular distance $[10,16,20,35]$. This implies

$$
\tau(T)=\tau_{0} \exp \left(\frac{C a^{2}}{\left\langle u^{2}\right\rangle(T)}\right),
$$

where $C$ is a constant. This result relates a larger MSD to a shorter alpha-relaxation time and it implies that the temperature dependence of the relaxation time is governed by the temperature dependence of the MSD. Hence the elastic model predicts that the change of the MSD just above $T_{g}$ is more dramatic the more fragile the liquid is. This agrees with the generally observed trend, although questions remain about at which time scale $\left\langle u^{2}\right\rangle$ should be considered. The glass-transition Lindemann criterion states that the $\left\langle u^{2}\right\rangle / a^{2}$, i.e., the relative vibrational amplitude of the atoms, at the glass transition should reach a certain universal value allowing diffusion on long time and length scales [10,34,36,37]. Recall that the Lindemann criterion is the rule that $\left\langle u^{2}\right\rangle / a^{2} \sim 1 \%$ when any crystal melts. If the glass transition is also characterized by such a universal number, there would be an appealing analog between crystal and glass "melting"-although the latter phenomenon is known to be cooling rate dependent.

In this paper we present a quantitative test of Eq. (1) based on MSD data in the nano- and picosecond time scale obtained by neutron backscattering and time-of-flight techniques on several molecular liquids, covering fragilities ranging from 49 to 161 . Three liquids were also studied under varying pressure. This allows one to examine different glass transitions of the same liquid, i.e., without changing the intermolecular interactions.

\section{EXPERIMENTAL}

The experiments were carried out on the back-scattering instruments IN10 and IN16 at the ILL. This spectrometers use the $\left(\begin{array}{lll}1 & 1 & 1\end{array}\right)$ reflection of Si-single crystals with a Bragg angle of $90^{\circ}$ as the monochromator and analyzers to reach an energy resolution of $\mathrm{FWHM}=1 \mu \mathrm{eV}$ (corresponding to a time scale of $\sim 4 \mathrm{~ns}$ ). The wavelength of the neutrons was $6.27 \AA$. The wave vector, $Q$, range covered was 0.2 to $1.9 \AA^{-1}$. The experiments were performed isobarically in cooling with a rate of $\sim 0.5 \mathrm{~K} / \mathrm{min}$. Pressure was applied using a clamp pressure cell mounted on the bottom of an insert to the cryostat. Sample transmission was $88 \%$ for the high-pressure measurements and $95 \%$ for the measurements at atmospheric pressure in standard aluminum cells. The liquids studied are: glycerol, cumene, dibuthyl-phthalate (DBP), m-toluidine, sorbitol, triphenylphosphite (TPP), and decahydroisoquinoline (DHIQ). The first three were also studied at elevated pressure $(300$ or $500 \mathrm{MPa})$; the pressure dependence of the glass-transition temperature was obtained from calorimetric experiments or extracted from dielectric spectroscopy under pressure. For glycerol a different pressure cell, dedicated for studying liquids under hydrostatic pressure, was used. This cell is built out of Niobium resulting in a low background and the pressure can be adjusted via a capillary from the outside of the cryostat, but the maximum pressure is limited to $300 \mathrm{MPa}$ [38]. The raw data correction was performed using the standard ILL software Sqwel which converts the measured data to the scattering law $S(Q, \omega)$ for the sample. The MSD is calculated from the measured elastic intensities by adopting the Gaussian approximation, $\ln (I)=A-\frac{Q^{2}\left\langle u^{2}\right\rangle}{3}$ with $A$ being a constant. The measured intensity is normalized to the intensity measured at low temperature, $T=4 \mathrm{~K}$, which means that the zero point motion is removed. We find that the $Q^{2}$ dependence is obeyed in the temperature range $0 \mathrm{~K}$ to $1.2 T_{g}[39,40]$. Even so, one should still be aware that the measured MSD can contain local relaxations which are unrelated to the vibrations and independent of the structural relaxation. 


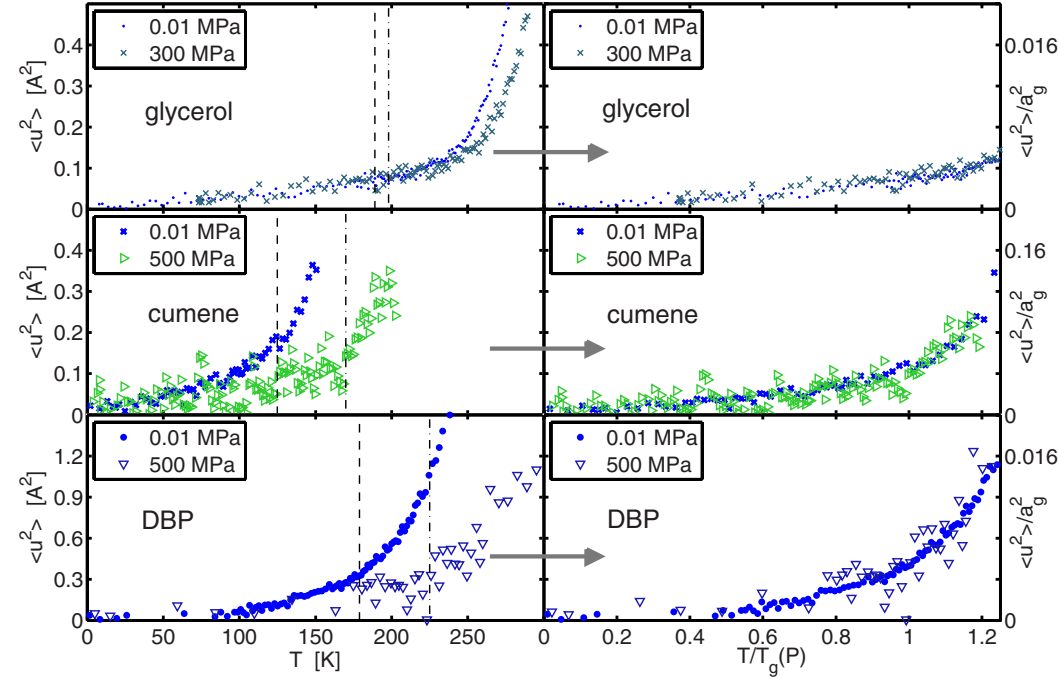

FIG. 1. (Color online) The MSD of glycerol, cumene and DBP at atmospheric pressure and at $500 \mathrm{MPa}$ (300 MPa for glycerol). The left hand side of the figure shows $\left\langle u^{2}\right\rangle$ and temperature on an absolute scale. The dashed lines indicate $T_{g}$, the dash-dotted lines $T_{g}$ at high pressure. The temperature scale in the right hand side of the figure is scaled by the pressure-dependent $T_{g}$ and the $y$-axis is scaled with $a^{2} \propto \rho^{-2 / 3}$ evaluated at $\left[T_{g}(P), P\right]$.
Supplementary experiments on DHIQ and DBP were carried out on the back-scattering instrument IN13 at atmospheric pressure. The energy resolution on IN13 is almost ten times wider, $\mathrm{FWHM}=8 \mu \mathrm{eV}$, meaning that the MSD we access in the measurement is probed on an almost ten times faster time scale $(\sim 0.5 \mathrm{~ns})$.

\section{LINDEMANN CRITERION}

How can the elastic-model prediction be tested? Comparing data for the same liquid at differing pressure, avoids making assumptions about the constant $C$ of Eq. (1). If it turns out that $C$ is common to all liquids, a universal (i.e., genuine) glass-transition Lindemann criterion is implied.

In Fig. 1 we present data for three liquids of different chemical nature, intermolecular interactions and fragility (glycerol, cumene, and dibutylphthalate). They are studied at ambient as well as at high pressures $(300 \mathrm{MPa}$ for glycerol, $500 \mathrm{MPa}$ for the two other liquids). The left part of each figure gives the mean-square displacement as a function of temperature at the two pressures where the dashed line marks the glass-transition temperature. The right part gives the data scaled as implied by Eq. (1). Thus the temperature is scaled by $T_{g}$ and the MSD by $a^{2}$, where we assume that the intermolecular distance scales with $a^{2} \propto \rho^{-2 / 3}$. The density, $\rho$ is evaluated at $\left(T_{g}(P), P\right)$ from known equations of state [41]. For all three liquids there is data collapse, showing that a Lindemann type criterion is fulfilled.

The scaling of the temperature axis is by far the most important for this data collapse in the pressure range studied. The estimated increase in density is less than $10 \%$. This gives a decrease of $a^{2}$ by approximately 5\%. This difference is almost indistinguishable in Fig. 1 due to the scatter of the data. A scaling with the pressure-dependent glass-transition temperature was earlier shown by two of us for a polymer sample in Ref. [38]. The earlier scaling is also done at relatively low pressures where the density does not change dramatically. It thus appears that for the systems studied so far, the MSD is constant along the glass-transition line in a P-Tdiagram, suggesting a Lindemann criterion. To verify whether the MSD is constant or, as suggested by the elastic model, whether the normalized MSD, $\left\langle u^{2}\right\rangle(T) / a^{2}$, is constant studies are needed in a larger pressure range. A constant value of $\left\langle u^{2}\right\rangle(T) / a^{2}$ with $a^{2} \propto \rho^{-2 / 3}$ along the glass-transition line, $\left(T_{g}(P), P\right)$, is also consistent with the existence of isomorphs as it is pointed out in Ref. [42]. Alternatively, the change of the intermolecular distance $a$ could be found based on a more local measure extracted from the static structure factor, $S(Q)$. However, measurements as a function of temperature and pressure show that the $Q$-dependence of the peak maximum follows the same behavior [43].

The next step is to investigate whether the constant $C$ in Eq. (1) is common to all liquids, as required by a universal glass-transition Lindemann criterion $[10,33]$. This is investigated in Fig. 2 by plotting $\left\langle u^{2}\right\rangle(T) / a^{2}$ as function of $T / T_{g}$ for a selection of liquids at ambient pressure with quite similar $T_{g}$ 's. If the constant $C$ were universal, $a^{2} /\left\langle u^{2}\right\rangle$ should be the same for all liquids at $T_{g}$. The figure shows that this is not the case since the number $\left\langle u^{2}\right\rangle\left(T_{g}\right) / a^{2}$ varies a factor of 3 going from glycerol to m-toluidine. It should be noted, though, that the temperature dependence of the MSD of m-toluidine has a strong increase far below $T_{g}$. This type of behavior has

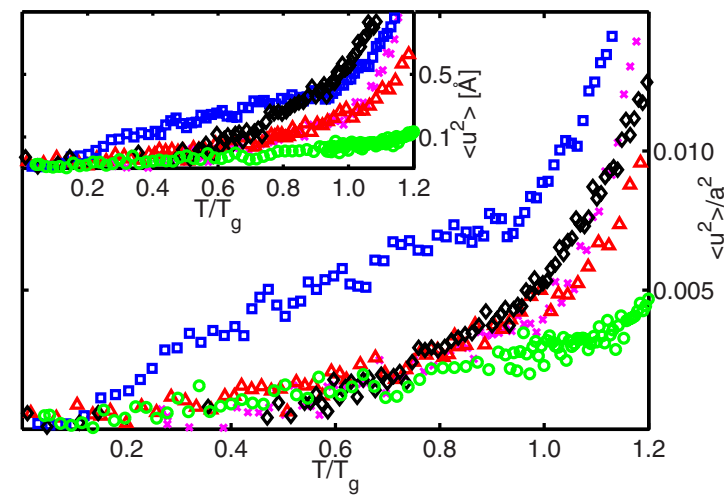

FIG. 2. (Color online) The temperature dependence of $\left\langle u^{2}\right\rangle$ scaled to $a^{2} \propto \rho^{-2 / 3}$ (see text for details) for 5 different liquids; glycerol (circles), DBP (diamonds), m-toluidine (squares), cumene (triangles), DHIQ (crosses). The temperature is scaled to $T_{g}$. The inset: the same data as in the main figure, here shown with the absolute value of $\left\langle u^{2}\right\rangle$. 


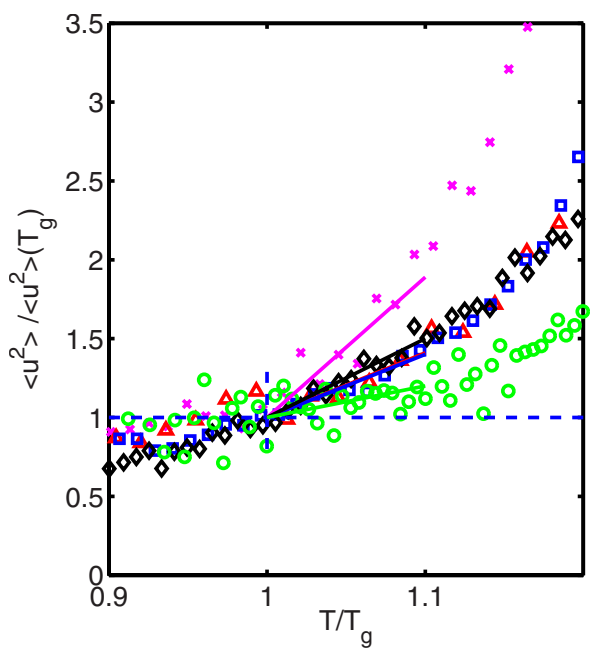

FIG. 3. (Color online) The measured $\left\langle u^{2}\right\rangle$ scaled to $\left\langle u^{2}\right\rangle_{T_{g}}$ for five different liquids. The temperature is scaled to $T_{g}$. glycerol (circles), DBP (diamonds), m-toluidine (squares), cumene (triangles), DHIQ (crosses). The lines above $T_{g}$ illustrate the fitted slopes used in Fig. 4.

earlier been seen in other systems and is associated with the methyl-group rotation [44]. Such type of local motion is probably independent of the glass-transition temperature and an irrelevant contribution to the apparent MSD with respect to the Lindemann criterion. Even for the four other liquids there is a factor of 2 in variation when comparing $\left\langle u^{2}\right\rangle\left(T_{g}\right) / a^{2}$. Based on these data and assuming that for DBP, DHIQ, and cumene the major contributions to the MSD are arising from displacements which are relevant for the structural relaxation near $T_{g}$, we cannot confirm the existence of a universal Lindemann criterion as predicted by Leporini and collaborators [33], at least not on the time scale we explore. The question is also discussed in a recent publication by the same group [45].

\section{IV. $T$ DEPENDENCE OF THE MSD ABOVE $T_{g}$}

We now apply a different scaling for the MSD by normalizing to the MSD at $T_{g}$. Figure 3 shows $\left\langle u^{2}\right\rangle(T) /\left\langle u^{2}\right\rangle_{T_{g}}$ as a function of $T / T_{g}$. The $\left\langle u^{2}\right\rangle$ value of the very fragile liquid DHIQ at the nanosecond rises most, the $\left\langle u^{2}\right\rangle$ of glycerol least, dramatically; the three remaining liquids, which all have similar intermediate fragilities, fall in between. The systems studied hence confirm the general trend that more fragile liquids have more temperature-dependent amplitude of the short-time MSD above $T_{g}$ than do less fragile [16]. The elastic models make a quantitative prediction regarding the relation between the temperature dependence of $\left\langle u^{2}\right\rangle$ and that of the alpha-relaxation time. Thus the elastic model leading to Eq. (1) is based on

$$
\frac{\Delta E(\rho, T)}{k_{B} T}=\frac{C a^{2}}{\left\langle u^{2}\right\rangle(\rho, T)} .
$$

Introducing the (isobaric) "activation energy index" [46], $I_{P}=-\left.\frac{d \ln \Delta E(T, \rho)}{d \ln T}\right|_{P}$, it follows that the elastic models predict

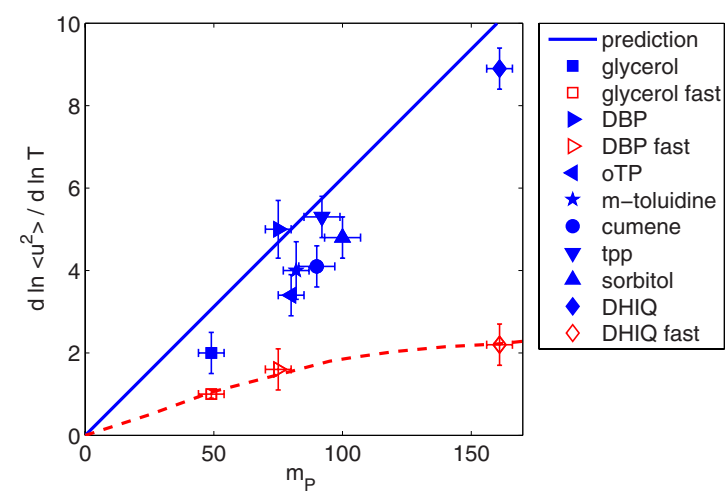

FIG. 4. (Color online) The value of $\left.\frac{\partial \ln \left\langle u^{2}\right\rangle}{\partial \ln T}\right|_{P}$ as a function of the isobaric fragility. Full symbols (blue) are data that refer to the nanosecond time scale ( $\sim \mathrm{ns}$ ), open symbols (red) are data obtained on IN13 on a ten times faster time scale $(\sim 0.4 \mathrm{~ns})$. The blue full line shows the expression Eq. (4) which follows from assuming $\Delta E(T) \propto T /\left\langle u^{2}\right\rangle$ as is predicted from the elastic model (using $\tau_{g}$ $=100 \mathrm{~s}$ and $\tau_{0}=10^{-14} \mathrm{~s}$ ). The red dashed line is a guide to the eye. Values and references are given in Table I. The points calculated at $\sim 4$ ns fall close to the predicted line, while the points calculated at $\sim 0.4$ ns where the $\left\langle u^{2}\right\rangle$ is less influenced by relaxations fall much below the line (the red dashed curve is a guide to the eyes). When points lie below the blue full line, the temperature dependence of $\left\langle u^{2}\right\rangle$ underestimates the temperature dependence of the activation energy. It should be noted that both $x$ and $y$ variables have considerable uncertainty as they are arrived at as numerical derivatives of data. The error bars on the literature data are our estimate based on experience and on the difference in reported values from different sources.

(where the weak temperature dependence of $a$ at constant pressure is ignored)

$$
I_{P}=-1+\left.\frac{d \ln \left\langle u^{2}\right\rangle}{d \ln T}\right|_{P} .
$$

Using the general relation [10] between the conventional fragility index and $I_{P}$ it follows that the model predicts a proportionality between Angell's (isobaric) fragility $m_{P}$ and the relative change of $\left\langle u^{2}\right\rangle$ with relative change in temperature,

$$
m_{P}=\log _{10}\left(\frac{\tau_{g}}{\tau_{0}}\right)\left(1+I_{P}\right)=\left.\log _{10}\left(\frac{\tau_{g}}{\tau_{0}}\right) \frac{d \ln \left\langle u^{2}\right\rangle}{d \ln T}\right|_{P},
$$

where $\tau_{0}=10^{-14} \mathrm{~s}$ is the microscopic time and $\tau_{g}=100 \mathrm{~s}$ is the relaxation time at the glass-transition temperature (where fragility is evaluated). Hence the elastic model predicts a correspondence between the slope seen in Fig. 2 at $T_{g}$ and the fragility found from the temperature dependence of the alpha-relaxation time.

Figure 4 tests this relation using fragilities and $T_{g}$ 's taken from literature (see Table I for values and references). The value of $\left.\frac{d \ln \left\langle u^{2}\right\rangle}{d \ln T}\right|_{P}\left(T=T_{g}\right)$ is in all cases calculated in the temperature range from $T_{g}$ to $\sim 1.1 T_{g}$, corresponding to the range where the fragility is determined. The data taken on the nanosecond time scale all lie close to the line. This result is rather convincing, especially because Eq. (4) not only predicts that there is a proportionality between $m_{P}$ and 
TABLE I. Values and references for the points shown in Fig. 4. The asterisk indicates that the value has been calculated from data in the paper.

\begin{tabular}{lccccc}
\hline \hline Compound & $m_{P}$ & Refs. & $\frac{d \ln \left\langle u^{2}\right\rangle}{d \ln T}$ & $\frac{d \ln \left\langle u^{2}\right\rangle}{d \ln T}$ fast & Refs. \\
\hline Glycerol & $40,53,54$ & {$[47],[48],[49]$} & 2 & 1 & This work [50] \\
DBP & 75 & {$[51]$} & 5 & 1.6 & This work \\
$o$-terphenyl (oTP) & $82,81,76,84$ & {$[47],[52],[53],[54]$} & $3.4^{*}$ & & {$[14]$} \\
$m$-toluidine & 79,84 & {$[55],[56]$} & 4 & & This work \\
Cumene & $90^{*}$ & {$[57]$} & 4.1 & & This work \\
TPP & $92^{*}$ & {$[58]$} & 6 & & This work \\
Sorbitol & 100 & {$[59]$} & 4.8 & & This work \\
DHIQ & $158,163^{*}$ & {$[60],[61]$} & 6 & 2.2 & This work \\
\hline \hline
\end{tabular}

$\left.\frac{d \ln \left\langle u^{2}\right\rangle}{d \ln T}\right|_{P}\left(T=T_{g}\right)$, but the value of the proportionality constant as well. The red points are take at shorter times, and are discussed in the section below.

\section{ROLE OF RELAXATIONS AND ANHARMONICITY}

Although Fig. 4 shows an overall agreement with the elastic-model prediction, when using the MSD on the nanosecond time scale, a number of issues remain to be considered. Not only, of course, is a more extensive study of different liquids needed, there are also other more fundamental issues. One problem is that in elastic models it is usually assumed that the measured $\left\langle u^{2}\right\rangle$ is purely vibrational, i.e., that no relaxational motion contributes to $\left\langle u^{2}\right\rangle$ around $T_{g}$. It is not likely that this assumption is generally correct, however. Thus we know from time-of-flight spectra that DHIQ has a strong quasielastic scattering already at $T_{g}$ [39]. Timeof-flight measurements have a broader resolution function, and consequently shorter time scale, so this quasielastic scattering corresponds to relaxation at even shorter times than the MSD probed by backscattering. The alpha relaxation itself also enters the experimental window at some time, possibly already when $\tau_{\alpha} \sim 1 \mu$ s if the relaxation function is very stretched. This happens intrinsically faster for fragile liquids than for strong liquids (for which, also, the relaxation functions are generally less stretched).

When considering relaxation it also appears that the finding of Fig. 4 is consistent with another phenomenological feature observed in the dynamic structure factor as measured from inelastic scattering. Namely, the observation that the relative strength of the boson peak compared to the fast relaxation, measured at $T_{g}$, is related to the isobaric fragility of the glass former: a parameter, $R$, is defined as the quasielastic intensity divided by the boson peak intensity, and proposed to increase with increasing isobaric fragility [24,27]. This model-independent assertion made by comparing the behavior of different glass formers is controversial [28], but appears more robust than many other correlations [29,39,62]. Our findings are clearly consistent with this observation, showing the importance of fast processes on a time scale of a few nanoseconds even close to $T_{g}$, where $R$ is determined for the most fragile liquids. This observation at the same time suggests that the boson peak intensity itself is not the rel- evant quantity for the correlation, but that it probably is the larger intensity of fast relaxation in fragile liquids that yields the correlation.

To investigate the role of relaxations further we have performed supplementary measurements of the MSD of DHIQ and DBP (for glycerol we used literature data [50] referring to the same time scale) using IN13 which has a broader than IN10 resolution and therefore accesses $\left\langle u^{2}\right\rangle$ on a time scale which is approximately ten times shorter. In Fig. 5 we compare the mean-square displacement of DHIQ found on the two different instruments, two distinct time scales. The measured $\left\langle u^{2}\right\rangle$ follow each other below $T_{g}$, which strongly indicates that we probe genuine vibrations in this regime and therefore that the finding of the Lindemann criterion is related to the vibrations, as predicted by the elastic models. Above $T_{g}$, on the other hand, we see a separation of the two curves. It is evident that the temperature dependence of the MSD on the nanosecond time scale probed by IN10 is much more pronounced than the temperature dependence on the shorter time scale probed by IN13. This dependence on the time scale indicates that we are not probing the purely vibra-

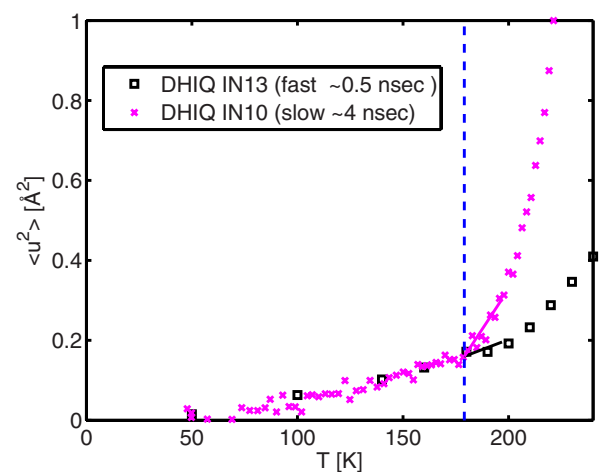

FIG. 5. (Color online) The MSD of DHIQ measured by IN13 (on a time scale of $\sim 0.4 \mathrm{~ns}$ ) and IN10 (on a time scale of $\sim 4 \mathrm{~ns}$ ). The dashed lines indicate $T_{g}$. The lines above $T_{g}$ illustrate the fitted slopes used in Fig. 4. The temperature dependence of the MSD is time scale independent below $T_{g}$ while it becomes strongly time scale dependent above $T_{g}$. This indicates that $\left\langle u^{2}\right\rangle$ below $T_{g}$ is dominated by vibrations while relaxations play a role on the nanosecond time scale in the liquid above $T_{g}$ (even close to 1 where the alpharelaxation time is still on the order of seconds). 
tional MSD on the nanosecond time scale, but rather a combination of vibration and fast relaxations.

To illuminate how the relaxations affect the value of $d \ln \left\langle u^{2}\right\rangle / d \ln T$ we adopt a simple "jump-diffusion" type modeling [63]: if on the time scale set by the experiment some molecules vibrate whereas others jump once or more, the MSD separates into two contributions: $\left\langle u^{2}\right\rangle=\left\langle u^{2}\right\rangle_{\text {vib }}+\left\langle u^{2}\right\rangle_{\text {jump. }}$ For the $\log$ - log derivatives one finds $d \ln \left\langle u^{2}\right\rangle / d \ln T$ $=A d \ln \left\langle u^{2}\right\rangle_{\text {vib }} / d \ln T+B d \ln \left\langle u^{2}\right\rangle_{\text {jump }} / d \ln T \quad$ where $\quad A$ $=\left\langle u^{2}\right\rangle_{\mathrm{vib}} /\left\langle u^{2}\right\rangle$ and $B=\left\langle u^{2}\right\rangle_{\text {jump }} /\left\langle u^{2}\right\rangle$ give the relative weights of the two contributions $(A+B=1)$. The jump contribution is most likely strongly temperature dependent. Thus any correction for this in order to get the pure elastic contribution to $d \ln \left\langle u^{2}\right\rangle / d \ln T$ pushes the points in Fig. 4 downward, i.e., further away from the line. This is exactly what we see for the $d \ln \left\langle u^{2}\right\rangle / d \ln T$ obtained from the IN13 data on DHIQ, which is also shown in Fig. 4. Similarly we see that the $d \ln \left\langle u^{2}\right\rangle / d \ln T$ calculated from glycerol data taken on IN13 reported by Wuttke [50] lie below the line. However, the difference between the two time scales is much less dominant for glycerol than for the very fragile DHIQ, indicating that the relaxation are more dominant in the latter.

Based on the above considerations, we conclude that using the vibrational part of the MSD as deviced by the elastic models [Eq. (1)] underestimates the temperature dependence of the activation energy. The reason for this could be that Eq. (1) is based on a simplified reasoning that basically ignores anharmonicities [10]. There are two nontrivial assumptions going into this reasoning: (a) the harmonic approximation, according to which the curvature at the minimum is inversely proportional to $\left\langle u^{2}\right\rangle$; (b) The energy barrier being proportional to the curvature at the minimum, as it would be if the potential were parabolic and scale accordingly. The first approximation applies to a good approximation at sufficiently low temperatures and may well apply to highly viscous liquids because these have fairly large energy barriers. The second approximation implies that if the barrier goes to zero, so does the curvature. However, this is not necessarily the case. Thus the simple elastic-model assumption that the barrier scales with curvature may break down. In summary, for a given temperature dependence of $\left\langle u^{2}\right\rangle$ a more realistic model might well predict larger $d \ln \Delta E / d \ln T$ than predicted by the elastic models. This corresponds to lowering the slope of the theoretical line of Fig. 4. This might explain why the $d \ln \left\langle u^{2}\right\rangle / d \ln T$ measured at short times where we expect vibrations to be dominant lie on the lower side of the line.

\section{DISCUSSION AND CONCLUSION}

The liquids studied show no universal glass-transition Lindemann criterion when we compare the MSD on the nanosecond time scale. Three of the liquids were studied at two different pressures. They obey a pressure-dependent Lin- demann criterion, as predicted by the elastic models. Thus the use of pressure reveals a connection which is probably masked by details in the molecular interactions and geometries when comparing different liquids; this suggests the existence of an intrinsic Lindemann criterion for each substance at their pressure-dependent glass transition. More extensive studies including several pressures along the glasstransition line of each liquid are needed in order to establish the range in which this result holds. A failure of a universal Lindemann criterion might also be due to the fact that relaxations contribute to the MSD as was discussed in the previous section. Similar to the elastic model the Lindemann criterion is based on a vibrational picture.

Above $T_{g}$ it appears that the elastic-model prediction underestimates the activation energy temperature dependence. We suggest that these deviations are caused by anharmonic effects. In this context it should be noted that despite the simple "harmonic" appearance of the elastic models, anharmonicities must play a role, even in the simplest elastic models. Thus the effective, temperature-dependent elastic constant (or curvature at energy minima) reflects anharmonicity because in truly harmonic potentials the elastic constants are temperature independent.

While the vibrational part of the mean-square displacement does not follow the prediction of the elastic models, we find that the total MSD measured, $\left\langle u^{2}\right\rangle(T)$, at the nanosecond time scale (vibrations and relaxations) approximately follows a proportionality of the type $\Delta E(T) \propto T /\left\langle u^{2}\right\rangle$, where $\Delta E(T)$ is the activation energy governing the alpha relaxation. This one-to-one finding is based on measurements of MSD on the nanosecond time scale by neutron scattering as function of temperature of molecular liquids covering a significant range of fragilities. The proportionality $\Delta E(T) \propto T /\left\langle u^{2}\right\rangle$ shows that there is a connection between the fast and the slow dynamics close to the glass transition. It is not clear how causal the relation is, whether the increase in MSD leads to higher mobility and consequently a speed up of the alpha relaxation, or the increased MSD is a due to a precursor of alpha relaxation itself, for example as a high frequency von Schweidler regime.

To summarize we find (i) an intrinsic Lindemann criterion for each liquid as predicted by the elastic models by studying the same liquids at different pressures; (ii) the temperature dependence of the MSD on the nanosecond time scale links to the liquid fragility as predicted by the elastic models. These observations are rationalized by introducing an anharmonicity in the elastic models, and they are fully consistent with other experimental features and correlations found in the literature. The findings in this work underline that a full understanding of the viscous slowing down must involve both the fast and the slow dynamics, and suggest that elastic models offer a starting point for understanding the connection between these different time scales. 
[1] G. Adam and J. H. Gibbs, J. Chem. Phys. 43, 139 (1965).

[2] E. Donth, J. Non-Cryst. Solids 53, 325 (1982).

[3] U. Tracht, M. Wilhelm, A. Heuer, H. Feng, K. Schmidt-Rohr, and H. W. Spiess, Phys. Rev. Lett. 81, 2727 (1998).

[4] L. Berthier, G. Biroli, J.-P. Bouchaud, L. Cipeletti, D. El Masri, D. L'Hôte et al., Science 310, 1797 (2005).

[5] C. Crauste-Thibierge, C. Brun, F. Ladieu, D. L'Hote, G. Biroli, and J. P. Bouchaud, Phys. Rev. Lett. 104, 165703 (2010).

[6] C. Dalle-Ferrier, C. Thibierge, C. Alba-Simionesco, L. Berthier, G. Biroli, J. P. Bouchaud, F. Ladieu, D. L'Hote, and G. Tarjus, Phys. Rev. E 76, 041510 (2007).

[7] A. Tobolsky, R. E. Powell, and H. Eyring, in Frontiers in Chemistry, edited by R. E. Burk and O. Grummit (Interscience, New York, 1943), Vol. 1, p. 125.

[8] S. V. Nemilov, Russ. J. Phys. Chem. 42, 726 (1968).

[9] J. C. Dyre, N. B. Olsen, and T. Christensen, Phys. Rev. B 53, 2171 (1996).

[10] J. C. Dyre, Rev. Mod. Phys. 78, 953 (2006).

[11] U. Buchenau and R. Zorn, EPL 18, 523 (1992).

[12] C. A. Angell, Science 267, 1924 (1995).

[13] T. Kanaya, I. Tsukushi, K. Kaji, J. Bartos, and J. Kristiak, Phys. Rev. E 60, 1906 (1999).

[14] R. Casalini and K. L. Ngai, J. Non-Cryst. Solids 293-295, 318 (2001).

[15] S. Magazu, G. Maisano, F. Migliardo, and C. Mondelli, Biophys. J. 86, 3241 (2004).

[16] K. L. Ngai, Philos. Mag. 84, 1341 (2004).

[17] K. L. Ngai, J. Non-Cryst. Solids 275, 7 (2000).

[18] E. Cornicchi, G. Onori, and A. Paciaroni, Phys. Rev. Lett. 95, 158104 (2005).

[19] M. Wyart, Phys. Rev. Lett. 104, 095901 (2010).

[20] R. W. Hall and P. G. Wolynes, J. Chem. Phys. 86, 2943 (1987).

[21] X. Xia and P. G. Wolynes, Proc. Natl. Acad. Sci. U.S.A. 97, 2990 (2000).

[22] V. Lubchenko and P. G. Wolynes, Annu. Rev. Phys. Chem. 58, 235 (2007).

[23] V. N. Novikov and A. P. Sokolov, Nature (London) 431, 961 (2004).

[24] V. N. Novikov, Y. Ding, and A. P. Sokolov, Phys. Rev. E 71, 061501 (2005).

[25] S. N. Yannopoulos and G. P. Johari, Nature (London) 442, E7 (2006).

[26] D. H. Torchinsky, J. A. Johnson, and K. A. Nelson, J. Chem. Phys. 130, 064502 (2009).

[27] A. P. Sokolov, E. Rössler, A. Kisliuk, and D. Quitmann, Phys. Rev. Lett. 71, 2062 (1993).

[28] S. N. Yannopoulos and G. N. Papatheodorou, Phys. Rev. B 62, 3728 (2000).

[29] K. Niss and C. Alba-Simionesco, Phys. Rev. B 74, 024205 (2006).

[30] T. Scopigno, G. Ruocco, F. Sette, and G. Monaco, Science 302, 849 (2003).

[31] T. Scopigno, D. Cangialosi, and G. Ruocco, Phys. Rev. B 81, 100202 (2010).

[32] A. Widmer-Cooper and P. Harrowell, J. Phys.: Condens. Matter $\mathbf{1 7}, \mathrm{S} 4025$ (2005).

[33] L. Larini, A. Ottochian, C. De Michele, and D. Leporini, Nat. Phys. 4, 42 (2008).

[34] D. S. Sanditov, Dokl. Phys. Chem. 390, 122 (2003).
[35] C. P. Flynn, Phys. Rev. 171, 682 (1968); A. P. Sokolov, A. Kisliuk, D. Quitmann, A. Kudlik, and E. Rössler, J. Non-Cryst. Solids 172-174, 138 (1994); C. M. Roland and K. L. Ngai, J. Chem. Phys. 104, 2967 (1996); M. M. Teeter, A. Yamano, B. Stec, and U. Mohanty, Proc. Natl. Acad. Sci. U.S.A. 98, 11242 (2001); F. W. Starr, S. Sastry, J. F. Douglas, and S. C. Glotzer, Phys. Rev. Lett. 89, 125501 (2002); P. Bordat, F. Affouard, M. Descamps, and K. L. Ngai, ibid. 93, 105502 (2004).

[36] U. Buchenau and A. Wischnewski, Phys. Rev. B 70, 092201 (2004).

[37] B. Frick, D. Richter, W. Petry, and U. Buchenau, Z. Phys. B: Condens. Matter 70, 73 (1988); V. K. Malinovsky and V. N. Novikov, J. Phys.: Condens. Matter 4, L139 (1992); A. Heuer and H. W. Spiess, J. Non-Cryst. Solids 176, 294 (1994); V. N. Novikov, E. Rössler, V. K. Malinovsky, and N. V. Surovtsev, EPL 35, 289 (1996); J. N. Onuchic, Z. Luthey-Schulten, and P. G. Wolynes, Annu. Rev. Phys. Chem. 48, 545 (1997); D. S. Sanditov, S. S. Sangadiev, and G. V. Kozlov, Glass Phys. Chem. 24, 539 (1998); V. N. Novikov and A. P. Sokolov, Phys. Rev. E 67, 031507 (2003); R. S. Berry and B. M. Smirnov, Phys. Usp. 48, 345 (2005).

[38] B. Frick and C. Alba-Simionesco, Physica B 266, 13 (1999).

[39] K. Niss, Ph.D. thesis, Université de Paris XI, 2007.

[40] C. Dalle-Ferrier, Ph.D. thesis, Université de Paris XI, 2009.

[41] The densities are obtained by extrapolation of literature data [57,64-66] using the well-known Tait equation and calculated expansion coefficients $[39,40]$.

[42] N. Gnan, T. B. Schrøder, U. R. Pedersen, N. P. Bailey, and J. C. Dyre, J. Chem. Phys. 131, 234504 (2009).

[43] C. Alba-Simionesco, D. Morineau, B. Frick, N. Higonenq, and H. Fujimori, J. Non-Cryst. Solids 235-237, 367 (1998).

[44] B. Frick and L. J. Fetters, Macromolecules 27, 974 (1994).

[45] A. Ottochian, C. De Michele, and D. Leporini, J. Chem. Phys. 131, 224517 (2009).

[46] J. C. Dyre and N. B. Olsen, Phys. Rev. E 69, 042501 (2004).

[47] C. Alba-Simionesco, A. Cailliaux, A. Alegria, and G. Tarjus, EPL 68, 58 (2004).

[48] N. O. Birge, Phys. Rev. B 34, 1631 (1986).

[49] M. Paluch, R. Casalini, S. Hensel-Bielowka, and C. M. Roland, J. Chem. Phys. 116, 9839 (2002).

[50] J. Wuttke, W. Petry, G. Coddens, and F. Fujara, Phys. Rev. E 52, 4026 (1995).

[51] K. Niss, C. Dalle-Ferrier, G. Tarjus, and C. Alba-Simionesco, J. Phys.: Condens. Matter 19, 076102 (2007).

[52] P. K. Dixon and S. R. Nagel, Phys. Rev. Lett. 61, 341 (1988).

[53] D. H. Huang and G. B. McKenna, J. Chem. Phys. 114, 5621 (2001).

[54] M. Paluch, K. L. Ngai, and S. Hensel-Bielowka, J. Chem. Phys. 114, 10872 (2001).

[55] C. Alba-Simionesco, J. Fan, and C. A. Angell, J. Chem. Phys. 110, 5262 (1999).

[56] A. Mandanici, M. Cutroni, and R. Richert, J. Chem. Phys. 122, 084508 (2005).

[57] A. J. Barlow, J. Lamb, and A. J. Matheson, Proc. R. Soc. London, Ser. A 292, 322 (1966).

[58] N. B. Olsen, T. Christensen, and J. C. Dyre, Phys. Rev. Lett. 86, 1271 (2001).

[59] N. B. Olsen, J. Non-Cryst. Solids 235-237, 399 (1998).

[60] R. Richert, K. Duvvuri, and L. T. Duong, J. Chem. Phys. 118, 1828 (2003). 
[61] R. Casalini, K. J. McGrath, and C. M. Roland, J. Non-Cryst. Solids 352, 4905 (2006)

[62] K. Niss, C. Dalle-Ferrier, V. M. Giordano, G. Monaco, B. Frick, and C. Alba-Simionesco, J. Chem. Phys. 129, 194513 (2008).
[63] K. S. Singwi and A. Sjolander, Phys. Rev. 119, 863 (1960).

[64] P. W. Bridgman, Proc. Am. Acad. Arts Sci. 67, 1 (1932).

[65] P. W. Bridgman, Proc. Am. Acad. Arts Sci. 77, 129 (1949).

[66] R. L. Cook, H. E. King, C. A. Herbst, and D. R. Herschbach, J. Chem. Phys. 100, 5178 (1994). 